\title{
Regressão aleatória Bayesiana para avaliação genética da resistência ao mal das folhas em seringueiras ${ }^{1}$
}

\author{
Bayesian random regression for genetic evaluation of South American leaf blight in \\ rubber trees
}

\author{
Victor Javier Cevallos Sandoval ${ }^{2}$, Fabyano Fonseca Silva ${ }^{3}$, Marcos Deon Vilela de Resende ${ }^{4}$, Leandro Roberto de \\ Macedo $^{5 *}$ e Paulo Roberto Cecon ${ }^{6}$
}

\begin{abstract}
RESUMO - O mal das folhas causado pelo fungo Microcyclus ulei é a doença mais séria dos seringais da América Latina. Com o objetivo de identificar clones de seringueira mais resistentes em diferentes ambientes ao longo do tempo, compararam-se diferentes modelos de regressão aleatória (MRA) ajustados via abordagem Bayesiana. Oito clones foram testados em campos clonais no delineamento em blocos completos casualizados com quatro repetições, utilizando-se 80 árvores por parcela. As duas fileiras centrais foram avaliadas a cada dois meses em relação às variáveis severidade (SEV) e índice de estroma em folha adulta (EFA). Foram incluídas como covariáveis nos modelos a circunferência do tronco e as variáveis climáticas de cada campo clonal. Os MRA foram comparados por meio do critério DIC (Deviance Information Criterion). O modelo M2 (que assumiu os efeitos aleatórios quadrático para clone e linear para planta) foi o melhor (menor valor de DIC) para descrever SEV e EFA em todas as localidades consideradas. Tal modelo permitiu inferir que os clones FDR 5788, CDC312 e CDC56 apresentaram maior resistência, enquanto o clone FX 3864 foi o que apresentou a maior suscetibilidade, em todas as localidades consideradas.
\end{abstract}

Palavras-chave: Hevea brasiliensis. Microcyclus ulei. Dados longitudinais. Inferência estatística. Simulação estocástica.

\begin{abstract}
This model enabled also to infer that the clones FDR 5788, CDC312 and CDC56 presented high resitance, while the colne FX 3864 was the most susceptible in all studied locals. South American leaf blight caused by the fungus Microcyclus ulei is the most serious disease of rubber plantations in Latin America. With the aim of identifying more resistant rubber-tree clones in various environments over time, different random regression models (MRA) adjusted via a Bayesian approach were compared. Eight clones were tested in clonal fields in a randomised complete block design with four replications, using 80 trees per lot. The two central rows were evaluated every two months for the variables severity (SEV) and stroma index of the adult leaf (EFA). Trunk circumference and the climatic variables of each clonal field were included in the models as covariates. The MRA were compared by the deviance information criterion (DIC). The M2 model (which assumed quadratic random effects for clones and linear effects for plants) was the best (lower values for DIC) in describing the SEV and EFA in all the locations being considered. Such a model suggests that the FDR 5788, CDC312 and CDC56 clones displayed greater resistance, while the FX 3864 clone displayed the highest susceptibility in all considered locations.
\end{abstract}

Key words: Hevea brasiliensis. Microcyclus ulei. Longitudinal data. Statistical Inference. Stochastic simulation.

\footnotetext{
DOI: $10.5935 / 1806-6690.20170017$

*Autor para correspondência

'Recebido para publicação em 23/11/2015; aprovado em 04/05/2016

Parte da Dissertação de Mestrado do primeiro autor apresentada ao Programa de Pós-Graduação em Genética e Melhoramento, Universidade Federal de Viçosa, Viçosa-MG, Brasil

${ }_{2}^{2}$ Instituto Nacional Autónomo de Investigações Agropecuárias/INIAP, Estación Experimental Santo Domingo, Km 38, La Concordia, Ecuador, vjcevallos75@yahoo.com

${ }^{3}$ Departamento de Zootecnia, Universidade Federal de Viçosa, Avenida Peter Henry Rolfs, s/n - Campus Universitário, Viçosa-MG, 36570-000

Viçosa-MG, Brasil, fabyanofonseca@ufv.br

${ }^{4}$ Embrapa Florestas, Estrada da Ribeira, Km 111, Colombo-PR, Brasil, 83.411-000, deon@ cnpf.embrapa.br

${ }^{5}$ Programa de Pós-Graduação em Estatística Aplicada e Biometria, Departamento de Estatística, Universidade Federal de Viçosa, Viçosa-MG

Brasil, leandro.macedo@ufv.br

${ }^{6}$ Departamento de Estatística, Universidade Federal de Viçosa, Viçosa-MG, Brasil, cecon@ufv.br
} 


\section{INTRODUÇÃO}

O mal das folhas da seringueira (Hevea brasiliensis L.), causado pelo fungo Microcyclus ulei, está presente em toda América Latina (LE GUEN et al., 2002, 2008; MATTOS et al., 2003), e ataca tanto árvores em estágio juvenil e também árvores que já atingiram a maturidade (FURTADO; MENTEN; PASSOS, 2008). Em árvores adultas, o ataque sucessivo pode causar o enfraquecimento das plantas, culminando em uma redução na produção de látex, e até conduzir à morte (GASPAROTTO et al., 1989).

A utilização de clones resistentes e produtivos é a medida mais eficiente de controle da doença. Estes são testados em ensaios clonais em grande escala considerando condições edafoclimáticas distintas de cada região produtora. As variáveis para determinar a resistência dos clones são a severidade do ataque (SEV) e o índice de estroma em folha adulta (EFA). Tais variáveis são medidas ao longo do tempo, geralmente a cada 60 dias, a fim de verificar o progresso da doença e sua dependência sazonal (RIVANO et al., 2010). Assim, os modelos estatísticos utilizados para identificar os clones mais eficientes devem contemplar a natureza longitudinal dos dados e sua relação com fatores genéticos e ambientais. Os modelos de regressão aleatória (MRA) satisfazem tais exigências, e são amplamente utilizados em melhoramento animal (PEIXOTO et al., 2014; PINHEIRO et al., 2013), porém ainda pouco explorados em melhoramento vegetal (MARIGUELE et al., 2011). Diferentemente dos modelos multicaracterísticos que fornecem predições de valores genéticos apenas nas idades observadas, os MRA permitem tal predição em qualquer ponto da trajetória longitudinal observada (RESENDE; REZENDE; FERNANDES, 2001).

Em estudos envolvendo dados longitudinais, a inferência Bayesiana tem sido utilizada com sucesso por reduzir o número de estimativas viesadas na presença de poucas observações e apresentar estimação por intervalo mais precisa em relação à obtida pela metodologia clássica (ROSA; GIANOLA; PADOVANI, 2004; ZHANG et al., 2007). Portanto, a abordagem Bayesiana de MRA apresenta-se como uma proposta interessante para a análise genética do desenvolvimento do mal das folhas em seringueiras.

Diante do exposto, objetivou-se apresentar uma abordagem Bayesiana para MRA contemplando simultaneamente a natureza longitudinal dos dados de desenvolvimento do mal das folhas em seringueira e aspectos genéticos e ambientais que possam influenciar tal desenvolvimento. Objetivou-se ainda testar diferentes MRA (graus polinomiais para efeitos genéticos e ambientais) bem como identificar clones mais resistentes em cada um dos ambientes considerados.

\section{MATERIAL E MÉTODOS}

Os campos clonais em grande escala foram estabelecidos na Estação Experimental Santo Domingo do Instituto Nacional Autónomo de Investigaciones Agropecuarias (INIAP) na província de Santo Domingo dos Tsáchilas $\left(00^{\circ} 01^{\prime} \mathrm{N}, 79^{\circ} 22^{\prime} \mathrm{W}\right.$ e altitude $\left.300 \mathrm{~m}\right)$, em fevereiro 2006; e na Estação de Agicom na província de Los Rios $\left(00^{\circ} 29^{\prime} \mathrm{S}, 79^{\circ} 21^{\prime}\right.$ 'W e altitude $\left.260 \mathrm{~m}\right)$, em março 2007.

Foram avaliados oito clones (meios-irmãos) de seringueira com quatro anos de idade (Tabela 1). Os ensaios foram estabelecidos em um delineamento em blocos completos casualizados com 4 repetições e 8 clones diferentes, utilizando-se 80 árvores por parcela, considerando uma área total de 5,9 ha. A unidade experimental foi de aproximadamente $1,568 \mathrm{~m}^{2}$, sendo as árvores plantadas em fileiras simples a $7 \mathrm{~m}$ de distância, com 2,8 m entre árvores e uma densidade de plantio de 510 árvores por hectare.

As observações fenotípicas foram efetuadas a cada dois meses (60 dias) no quarto ano de idade das plantas, nas duas fileiras centrais, isto é, $50 \%$ do número total de plantas de cada parcela. A severidade (SEV) da doença foi avaliada por meio da porcentagem de área foliar lesionada seguindo uma escala diagramática (GASPAROTTO et al., 1989) de 1 a 5 (1: de 0 a $0,8 \%$; 2: de 1 a 5\%; 3: de 6 a 15\%; 4: de 18 a 30\%; e 5: de 40 a $100 \%$ de área foliar lesionada). Os estromas são estruturas fúngicas no lado superior da folha que contém ascósporos, de forma que sua densidade é um bom indicador da capacidade de um inóculo para formar um teliósporo (esporos de resistência do fungo). $\mathrm{O}$ índice de estroma em folha adulta (EFA) foi quantificado por meio da seguinte escala semiquantitativa variando de 0 a 4 , em que os valores $0 ; 1 ; 2 ; 3$ e 4 , indicaram, respectivamente, ausência de estromas, de 1 a 5 , de 6 a 10 , de 11 a 30 e mais de 30 estromas por folíolo.

Os MRA geralmente incluem um polinômio para representar as trajetórias longitudinais dos efeitos fixos e aleatórios (GILMOUR, 2006; MEYER; KIRKPATRICK, 2005), e sua grande vantagem é possibilitar a obtenção de estruturas de covariâncias entre os diferentes tempos através de funções de covariância para os efeitos aleatórios de interesse, sendo estas obtidas de matrizes de covariância entre os coeficientes de regressão dos polinômios em questão. No presente estudo, o seguinte modelo foi considerado: 
Tabela 1 - Descricão dos clones de seringueira testados, bem como seus parentais, país de origem e ano de introdução

\begin{tabular}{lccc}
\hline \multicolumn{1}{c}{ Clone } & Parentesco (parental 1 x parental 2) & País de origem & Ano de introdução \\
\hline FX 3864 & PB 86 x FB38 & Brasil & 1964 \\
FX 4098 & PB 86 x B110 & Brasil & 2004 \\
CDC 56 & MDX 91 x RRIM 614 & Guatemala & 2004 \\
CDC 312 & AVROS 308 x MDX 40 & Guatemala & 2004 \\
FDR 4575 & FDR 18 x FX 3032 & Brasil & 2004 \\
FDR5597 & HAR 68 x TU 42-525 & Brasil & 2004 \\
FDR 5788 & HAR 8 x MDF 180 & Brasil & 2004 \\
MDF 180 & Clone primario de H. brasiliensis & Peru & 2004 \\
\hline
\end{tabular}

$$
Y_{i j k l}=\sum_{m=0}^{k_{\mu}} \mu_{m} t_{k}+\sum_{m=0}^{k_{c}} c_{j m} t_{j k}+\sum_{m=0}^{k_{p}} p_{i m} t_{i k}+b_{k}+e_{i j k}
$$

em que: $y_{i j k l}$ é o valor das variáveis analisadas (SEV e EFA) da planta $i(\mathrm{i}=1 ; 2 ; \ldots ; 40)$ do clone $j(\mathrm{j}=1 ; 2 ; \ldots ; 8)$ avaliada no tempo $(t) \mathrm{k}(\mathrm{k}=1 ; 2 ; \ldots ; 6)$ dentro do bloco 1 $(1=1 ; 2 ; \ldots ; 4) ; \mu_{\mathrm{m}}$ é o coeficiente de regressão médio da população; $c_{j m}$ e $p_{i m}$ são, respectivamente, os coeficientes de regressão aleatória dos efeitos de clone e planta; $\mathrm{k}_{\mu}$, $k_{c}$ e $k_{p}$ correspondem, respectivamente, aos graus dos polinômios da curva média da população e dos efeitos de clone e planta; b é o efeito de blocos; e e $\mathrm{i}_{\mathrm{ijk}}$ é o termo de erro aleatório.

Em notação matricial, o modelo (1) é dado por:

$y=z_{\mu} \mu+z_{c} c+z_{p} p+z_{b} b+e$,

em que: $y$ é o vetor de observações longitudinais individuais, $\mu$ é o vetor de dimensão $\left(\mathrm{k}_{\mu}+1\right)$ de coeficientes médios da população; $c$ é o vetor de dimensão $\left(\mathrm{k}_{\mathrm{c}}+1\right) * 8$ de coeficientes de regressão aleatória dos efeitos de clone, $p$ é o vetor de dimensão $\left(\mathrm{k}_{\mathrm{p}}+1\right) * 40$ de coeficientes de regressão aleatória dos efeitos de planta; $b$ é o efeito de blocos; $Z_{\mu}$, $Z_{c}$ e $Z_{p}$ são, respectivamente, matrizes de incidência para os coeficientes polinomiais representativos dos efeitos de média, clone e planta, $Z_{b}$ é a matriz de incidência do efeito de bloco; $e$ é o vetor de resíduos.

Sob a ótica bayesiana, assume-se uma distribuição conjunta para os dados amostrais, denominada de função de verossimilhança, e também distribuições de probabilidade para os parâmetros de interesse, denominadas de distribuições a priori, a fim de obter a distribuição a posteriori por meio do produto destas duas distribuições já mencionadas (SORENSEN; GIANOLA, 2002). No presente estudo, assumiu-se uma distribuição normal para os dados amostrais:y N $\left(Z_{\mu} \mu+Z_{c} c+Z_{p} p+Z_{b} b\right.$, $\left.\mathrm{I}^{2}\right)$, uma vez que o teste Shapiro-Wilk ao nível de $5 \%$ de significância indicou normalidade dos dados para todas as características em todos os locais avaliados, e as distribuições a priori associadas aos coeficientes médios da população foram não-informativas. Já as distribuições a priori para os coeficientes de regressão aleatória foram: $\mathrm{c} \sim \mathrm{N}\left(0, \mathrm{~A} \otimes \mathrm{K}_{\mathrm{c}}\right)$, sendo $\mathrm{A}$ a matriz de parentesco entre os clones (obtida por meio das informações da Tabela 1) e $\mathrm{K}_{\mathrm{c}}$ uma matriz de dimensão $\left(\mathrm{k}_{\mathrm{c}}+1\right) \mathrm{x}\left(\mathrm{k}_{\mathrm{c}}+1\right)$ de covariâncias entre coeficientes de regressão dos diferentes graus para os efeitos de clone; $\mathrm{p} \sim \mathrm{N}\left(0, \mathrm{I}_{\mathrm{n}} \otimes \mathrm{K}_{\mathrm{p}}\right)$, sendo $\mathrm{I}_{\mathrm{n}}$ uma matriz identidade de ordem $n$ e $\mathrm{K}_{\mathrm{p}}$ uma matriz de dimensão $\left(\mathrm{k}_{\mathrm{p}}+1\right) \times\left(\mathrm{k}_{\mathrm{p}}+1\right)$ de covariâncias entre coeficientes de diferentes graus para os efeitos de planta. Para o efeito aleatório de blocos e residual, as seguintes distribuições foram assumidas: $b \sim \mathrm{N}\left(0, \mathrm{I} \sigma_{b}^{2}\right)$ e e $\sim \mathrm{N}\left(0, \mathrm{I} \sigma^{2}\right)$.

Considerando as matrizes de covariância $K_{c}$ e $\mathrm{K}_{\mathrm{p}}$ como desconhecidas, bem como as variâncias de blocos e residual, há a necessidade de se atribuir também distribuições a priori para estes parâmetros. Assim, para as matrizes de covariâncias foram assumidas distribuições de Wishart invertidas e para os componentes de variância distribuições qui-quadrado invertidas tal como em Meyer (2005) e Bignardi et al. (2012).

Uma vez que é possível considerar diferentes modelos alterando apenas o grau polinomial de cada efeito, o critério DIC (Deviance Information Criterion) foi utilizado para comparar tais modelos, de modo que quanto menor o valor do DIC, melhor o modelo (SPIEGELHALTER et al., 2002). Após sucessivas análises-piloto, quatro modelos foram previamente e ajustados às duas variáveis (SEV e EFA) nos dois ambientes (INIAP e Agicom). Nestes modelos a curva média foi linear, havendo alteração apenas nos graus polinomiais dos efeitos de clone e planta. Assim, o modelo 1 (M1) foi definido como efeito linear para clone e linear para planta, o modelo 2 (M2) como efeito quadrático para clone e linear para planta, o modelo 3 (M3) como efeito 
linear para clone e quadrático para planta, e o modelo 4 (M4) como efeito quadrático para clone e para planta.

Os MRA foram implementados por meio do pacote MCMCglmm do software R (R Core Team, 2015). Foram consideradas 100.000 iterações no algoritmo Gibbs sampler, que é um procedimento de simulação estocástica, com descarte das 50.000 iniciais e intervalo de amostragem de 5 iterações, resultando em cadeias de 10.000 iterações. As estimativas dos parâmetros utilizadas para a confecção dos gráficos contemplando o valor genético dos clones ao longo do tempo foram consideradas como sendo a média a posteriori das cadeias mencionadas.

\section{RESULTADOS E DISCUSSÃO}

Na Figura 1 são apresentados os valores de DIC para cada modelo avaliado (M1, M2, M3 e M4) nos dois ambientes considerados, INIAP (Figura 1A) e Agicom (Figura 1B). O modelo M2 (efeitos aleatórios quadráticos para clone, e linear para planta) foi o melhor para descrever as características SEV e EFA nas duas localidades por ter apresentado o menor valor de DIC. Optou-se pelo critério DIC por este ser indicado para fazer a comparação entre modelos complexos, principalmente quando os mesmos apresentam diferentes números de parâmetros (SPIEGELHALTER et al., 2002), tal como os MRA com diferentes graus polinomiais conforme abordado no presente estudo.

Observou-se na Figura 2 que no ambiente INIAP os clones FX3864 (testemunha) e FX4098 apresentaram as retas mais elevadas para SEV e EFA, sendo então aqueles mais susceptíveis sob o ponto de vista genético.
Já os clones FDR5788 e CDC 312 apresentaram valores genéticos abaixo do zero com tendência decrescente para SEV e EFA, indicando que estes clones são mais tolerantes e possuem melhores adaptações ao ambiente. Os clones que apresentaram a menor nota de ataque e índice de estroma foram CDC312, CDC56, MDF180, FDR4575 e FDR597, dos quais, os clones CDC312 e CDC56 expressaram características genotípicas muito similares para SEV, possivelmente por serem meios irmãos, e desfrutarem parte da mesma constituição genética. Estes apresentaram praticamente o mesmo valor genético para SEV, cuja reta foi abaixo de zero e com tendência decrescente. Porém, para EFA, os valores genéticos do clone CDC56 se mantiveram constantes, enquanto um declínio foi observado para CDC312. Em resumo, os melhores clones para o ambiente INIAP com base no valor genético para SEV foram os FDR5788, CDC 312 e CDC 56, enquanto que para EFA foram os FDR5788 e CDC312.

No ambiente Agicom (Figura 3), similarmente ao observado no ambiente INIAP, notou-se que os clones FX3864 (testemunha) e FX4098 apresentaram maior susceptibilidade genética ao mal das folhas. Os clones FDR5788, CDC312, CDC56, MDF180, FDR4575 e FDR5597 apresentaram valores genéticos para SEV e EFA similares para ambas as características, e devido ao efeito de ambiente, menor quantidade de chuva da localidade, o que limita o desenvolvimento do fungo, o valor genético deles também foi expresso em torno de zero com tendência decrescente. Embora tenham apresentado comportamentos genéticos similares no ambiente em questão, pode-se inferir que os melhores clones com base nos valores genéticos para SEV foram MDF180, CDC312, CDC56, FDR4575, FDR5597 e FDR 5788; enquanto que para EFA foram MDF180, CDC312, CDC56, FDR4575, FDR5597, FDR5788 e FDR4098.

Figura 1 - Valores do critério DIC para comparação Bayesiana dos modelos (M1, M2, M3 e M4) em cada ambiente, INIAP (A) e Agicom (B), para as características severidade (SEV) e índice de estroma em folha adulta (EFA) relacionadas ao mal da folha em seringueiras
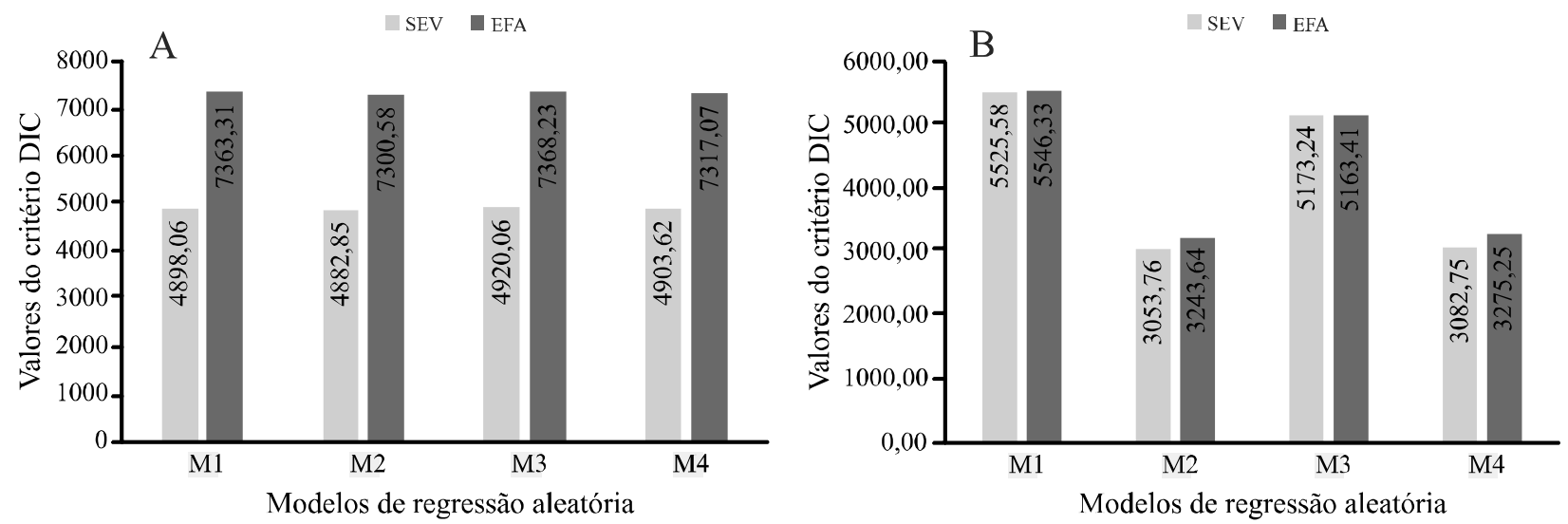
Figura 2 - Observações fenotípicas (pontos) e valores genéticos estimados (retas) para oito clones de seringueiras cultivados na localidade INIAP e avaliados para as características severidade (SEV) (A) e índice de estroma em folha adulta (EFA) (B)
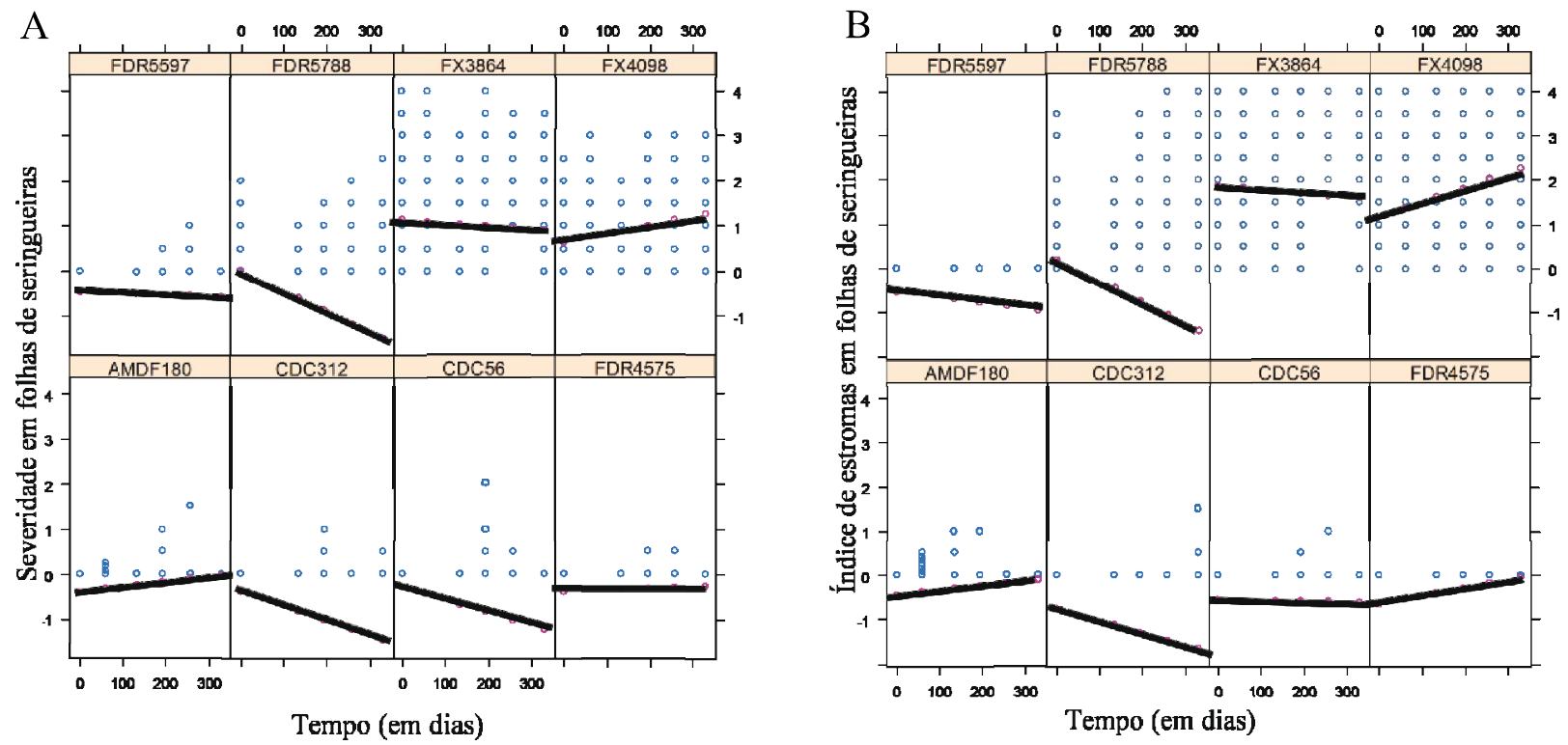

Figura 3 - Observações fenotípicas (pontos) e valores genéticos estimados (retas) para oito clones de seringueiras cultivados na localidade Agicom e avaliados para as características severidade (SEV) (A) e índice de estroma em folha adulta (EFA) (B)

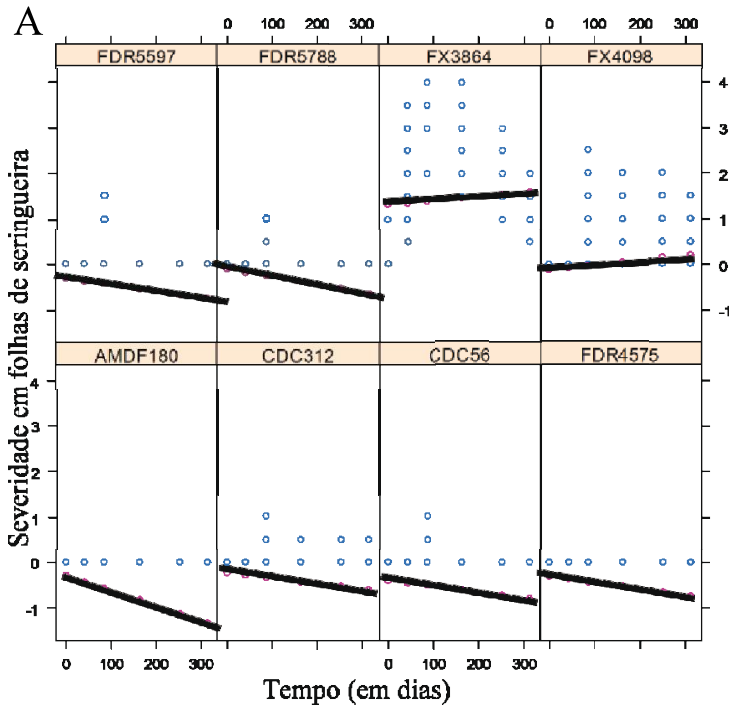

O presente estudo permitiu inferir que na localidade INIAP a intensidade da doença causada pelo fungo M. ulei foi maior do que na localidade Agicom, uma vez que a primeira apresenta condições climáticas mais favoráveis, maior índice pluviométrico, à ocorrência do mal da folha da seringueira conforme reportado

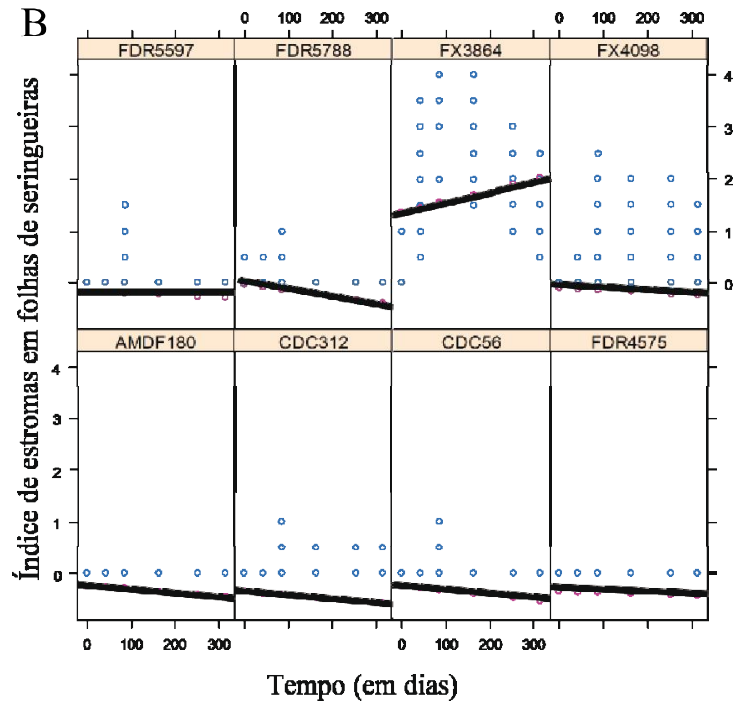

por Gasparotto et al. (1989). Estes autores também demonstraram a importância do molhamento foliar para germinação dos esporos e para estabelecimento das infecções de vários fitopatógenos, e reportou que a $24{ }^{\circ} \mathrm{C}$ ocorreram infecções com apenas seis horas de molhamento foliar. 
O clone FX3864 (testemunha) foi o que apresentou a maior suscetibilidade genética nas duas localidades, principalmente maior severidade de ataque e estromas na localidade INIAP. Vários autores verificaram que este clone foi muito susceptível em condições climáticas parecidas (JAIMES; ROJAS, 2011), e destacou-se um ataque severo em um ensaio feito em Magdalena Médio na Colômbia, (RIVANO et al., 2013). Já o clone FDR5788 mostrou menor incidência da doença na localidade INIAPe Agicom, mas outros clones tais como FDR5597, MDF180, CDC312, CDC 6 e FDR4575 mostraram-se resistentes a doença nas duas localidades, inclusive já foram testados na Bahia (nordeste brasileiro) onde apresentaram melhores níveis de resistência parcial (RIVANO et al., 2013).

\section{CONCLUSÕES}

1.O modelo com efeitos aleatórios quadrático para clone e linear para planta é o que melhor descreve as características SEV e EFA em todas as localidades;

2. Os clones FDR5788, CDC312 e CDC56 foram os mais resistentes ao mal da folha da seringueira, e o clone FX3864 o mais susceptível.

\section{REFERÊNCIAS}

BIGNARDI, A. et al. Bayesian analysis of random regression models using B-splines to model test-day milk yield of Holstein cattle in Brazil. Livestock Science, v. 150, p. 401-406, 2012.

FURTADO, E. L.; MENTEN, J. O. M.; PASSOS, R. Intensidade do Mal das Folhas em plantas jovens e adultas de seis clones de seringueira na região do Vale do Ribeira. Tropical Plant Pathology, v. 33, n. 2, p. 130-137, 2008.

GASPAROTTO, L. et al. Epidemiologia do mal das folhas da seringueira. I- Ponte Nova-MG. Fitopatologia Brasileira, v. 14, n. 1, p. 65-70, 1989.

GILMOUR, A. R. Statistical models for multidimensional (longitudinal/spatial) data. In: WORLD CONGRESS ON GENETICS APPLIED TO LIVESTOCK PRODUCTION, 8., 2006, Belo Horizonte. Procedings... Belo Horizonte, 2006. Disponível em: <http://www.wcgalp8.org.br/wcgalp8/articles/ paper/25_339-773.pdf>. Acesso em: 5 ago. 2015.

JAIMES, S. Y. Y.; ROJAS, M. J. Enfermedades foliares del caucho (Hevea brasiliensis Muell. Arg.) establecido en un campo clonal ubicado en el Magdalena Medio Santandereano (Colombia). Corpoica Ciencia y Tecnologia Agropecuaria, v. 12, n. 1, p. 65-76, 2011.

LE GUEN, V. et al. Evaluation of field resistance to Microcyclus ulei of a collection of Amazonian rubber tree (Hevea brasiliensis) germplasm. Crop Breeding and Applied Biotechnology, v. 2, n. 1, p. 141-148, 2002.
LE GUEN, V. et al. Long lasting rubber tree resistance to Microcyclus ulei characterized by reduced conidial emission and absence of teleomorph. Crop Protection, v. 27, n. 12, p. 1498-1503, 2008.

MARIGUELE, K. H. et al. Métodos de análise de dados longitudinais para o melhoramento genético da pinha. Pesquisa Agropecuária Brasileira, v. 46, n. 12, p. 1657-1664, 2011.

MATTOS, C. et al. Variabilidade de Isolados de Microcyclus ulei no Sudeste da Bahia. Fitopatologia Brasileira, v. 28, n. 5, p. 502-507, 2003.

MEYER, K. Advance in methodology for random regression analyses. Australian Journal of Experimental Agriculture, v. 45 , n. 8 , p. $847-858,2005$.

MEYER, K.; KIRKPATRICK, M. Restricted maximum likelihood estimation of genetic principal components and smoothed covariance matrices. Genetics Selection Evolution, v. 37, n. 1, p. 1-30, 2005.

PEIXOTO, M. G .C. D. et al. Random regression models to estimate genetic parameters for milk production of guzerat cows using orthogonal Legendre polynomials. Pesquisa Agropecuária Brasileira, v. 49, n. 5, p. 373-383, 2014.

PINHEIRO, V. R. et al. Mapeamento de QTL para características de crescimento de suínos por meio de modelos de regressão aleatória. Pesquisa Agropecuária Brasileira, v. 48, n. 2, p. 190-196, 2013.

R CORE TEAM. R: a language and environment for statistical computing. Vienna: R Foundation for Statistical Computing, 2015. Disponível em: <http://www.r-project.org/>. Acesso em: 14 set. 2015 .

RESENDE, M. D. V.; REZENDE, G. D. S. P.; FERNANDES, J. S. C. Regressão aleatória e funções de covariância na análise de medidas repetidas. Revista Brasileira de Matematica e Estatística, v. 19, n. 1, p. 21-40, 2001.

RIVANO, F. et al. Assessing resistance off rubber tree clones to Microcyclus ulei in large-scale clones trials in Ecuador: a less time-consuming field method. European Journal of Plant Pathology, v. 126, n. 4, p. 541-552, 2010.

RIVANO, F. et al. Breeding Hevea brasiliensis for yield, growth and SALB resistance for high disease environments. Industrial Crops and Products, v. 44, n. 12, p. 659-670, 2013.

ROSA, G. J. M.; GIANOLA, D.; PADOVANI, C. R. Bayesian longitudinal data analysis with mixed models and thick-tailed distributions using MCMC. Journal of Applied Statistics, v. 31, n. 7, p. 855-873, 2004.

SORENSEN, D.; GIANOLA, D. Likelihood, Bayesian and MCMC methods in quantitative genetics. 1st ed. New York: Springer-Verlag, 2002. $740 \mathrm{p}$.

SPIEGELHALTER, D. J. et al. Bayesian measures of model complexity and fit. Journal of the Royal Statistical Society: Series B (Statistical Methodology), v. 64, n. 4, p. 583-639, 2002.

ZHANG, Z. et al. Bayesian analysis of longitudinal data using growth curve models. International Journal of Behavioral Development, v. 31, n. 4, p. 374-383, 2007. 